IdeAs

Idées d'Amériques

$10 \mid 2017$

États-Unis / Cuba : une nouvelle donne?

\title{
Le Brésil des réformes du travail et l'institutionnalisation de la précarité
}

Sayonara Grillo Coutinho Leonardo da Silva

Traducteur : Christian Azaïs

OpenEdition

Journals

Édition électronique

URL : https://journals.openedition.org/ideas/2224

DOI : $10.4000 /$ ideas.2224

ISSN : 1950-5701

Éditeur

Institut des Amériques

Référence électronique

Sayonara Grillo Coutinho Leonardo da Silva, «Le Brésil des réformes du travail et l'institutionnalisation de la précarité », IdeAs [En ligne], 10 | 2017, mis en ligne le 19 décembre 2017, consulté le 18 octobre 2022. URL : http://journals.openedition.org/ideas/2224 ; DOI : https://doi.org/10.4000/ideas.2224

Ce document a été généré automatiquement le 18 octobre 2022

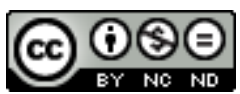

Creative Commons - Attribution - Pas d'Utilisation Commerciale - Pas de Modification 4.0 International - CC BY-NC-ND 4.0

https://creativecommons.org/licenses/by-nc-nd/4.0/ 


\title{
Le Brésil des réformes du travail et l'institutionnalisation de la précarité
}

\author{
Sayonara Grillo Coutinho Leonardo da Silva \\ Traduction : Christian Azaïs
}

1 Dans le contexte de grave crise politique, éthique et institutionnelle, les politiques du travail, de la protection sociale et des droits humains, construites pendant les décennies de consolidation démocratique au Brésil, sont mises en échec. Sous l'effet de politiques économiques ultralibérales, des demandes interminables des entrepreneurs et du vieux clientélisme entre le pouvoir exécutif et les secteurs parlementaires, l'année 2017 restera gravée dans l'histoire des relations professionnelles brésiliennes. Le retour des manifestations syndicales et populaires contre les réformes annoncées de la sécurité sociale ${ }^{1}$ et du travail, contre le gel des finances publiques et l'institution d'un plafond de dépenses - malgré la déflagration de la plus grande grève générale - n'ont pas été capables de contenir ou d'atténuer la déprédation rapide et intense des institutions publiques du travail et de ses systèmes de régulations étatiques et syndicales - le droit du travail et la négociation collective et leurs principaux acteurs syndicaux et agents publics.

2 La Consolidation des lois du travail ${ }^{2}$ est déformée par la réforme de plus d'une centaine de dispositifs, approuvée par la loi $\mathrm{n}^{\circ} 13467$ du 13 juillet 2017, qui ébranlent les fondements du système national de relations professionnelles, construit dans la perspective d'une démarchandisation du travail (Esping Andersen G., 1991 : 85-116).

3 La vague réformatrice ne s'arrête pas là. Elle se manifeste dans un processus continu de reformulation de la loi qui, entre autres, élargit le travail temporaire et les formes d'externalisation des services (loi 13429/2017), gèle les salaires de nombre d'agents de l'Etat en réduisant leurs valeurs nominales par l'augmentation des cotisations à la sécurité sociale ${ }^{3}$.

4 Même le concept de "travail analogue à de l'esclavage » a été substantiellement limité la semaine qui a précédé une nouvelle séance parlementaire où serait analysée la 
demande d'autorisation pour accuser le président Temer de corruption (Arrêté MTb 1129/2017). L'illégalité de cet arrêté, qui en finit pratiquement avec les politiques de sécurité sociale, réparatrices et répressives envers le travail esclave, dans un pays qui a été récemment condamné par la Cour interaméricaine des droits humains pour son omission et sa connivence vis-à-vis des pratiques d'esclavage contemporain sur son territoire, est l'expression la plus éloquente de la régression sociale vécue. En raison de son poids symbolique et de sa gravité, le STF (Suprême tribunal fédéral) a prononcé la suspension de ses effets.

5 Les arguments du gouvernement brésilien en faveur de la réforme du travail sont éculés. Sans base théorique ou empirique, il s'agit de politiques fondées sur des a priori idéologiques selon lesquels la flexibilité du travail conduirait à une diminution du chômage, à l'amélioration du marché du travail, à des augmentations de salaire et de la productivité, au dépassement de la crise économique, à la réduction du nombre d'actions judiciaires en matière de travail et à l'augmentation de la compétitivité nationale.

6 Les projections gouvernementales relatives à la productivité n'ont pas été bien expliquées. Comment le faire sans relier les indicateurs qui soutiennent son accroissement et qui en même temps ne prennent pas en compte les facteurs négatifs qui poussent vers le bas les taux nationaux? La formation et la qualification professionnelle, l'innovation de processus et les technologies, la flexibilité sans précarité, les investissements publics et privés, les politiques de valorisation des ressources humaines sont quelques-unes des variables qui ont un effet positif sur le taux de productivité. Pourtant, elles ne sont pas inscrites à l'agenda gouvernemental centré sur des réformes dont les conséquences comme l'augmentation du taux déjà élevé d'accidents du travail a une incidence sur l'accroissement de la précarité au travail et des effets négatifs sur les comptes publics de la sécurité sociale, choses amplement connues.

7 Avec l'introduction d'une multiplicité de contrats de travail qui stimulent le turn-over, éloignent le travailleur de la vie de l'entreprise, l'empêchant de mener à bien son projet de vie et affectant négativement sa subjectivité et son projet de qualification à moyen ou long terme, il convient de se demander si cette réforme pourra contribuer à augmenter la compétitivité des entreprises et la compétitivité nationale. Il nous semble que non. Il en est de même des formules qui rendent à l'entrepreneur son ample liberté pour déterminer quand, comment et combien doit-on travailler, autant de thèmes qui font partie du vocabulaire officiel. La reformulation prévue dans la loi 13467/2017 contient des dispositifs qui limitent l'accès des travailleurs à la justice du travail, comme le paiement des coûts de la procédure, des honoraires, une gratuité de la justice plus restreinte, autant de charges financières lourdes pour les requérants qui dans la plupart des cas sont pauvres. Ces règles ont vu leur constitutionnalité questionnée par le Procureur général de la République devant le STF (ADI - Action directe d'inconstitutionnalité - $\mathrm{n}^{\circ} 7566$ ).

8 Au Brésil, dans de nombreux secteurs, domine la logique de la rupture du contrat sans motif, sans paiement de salaire ni d'indemnités de rescision. La conséquence de ce nonrespect fréquent et délibéré des normes est la forte judiciarisation des relations professionnelles. Mais à une époque de post-réforme, si jamais les requérants qui " courent après leurs droits " auprès de la Justice du travail arrivent à dépasser les barrières économiques et de procédure, ils trouveront en face d'eux un appareil 
judiciaire fragilisé par des règles qui cherchent à maintenir la magistrature du travail dans le régime napoléonien dépassé du juge "bouche de la loi», en raison de dispositions qui tentent de stopper le contenu des normes collectivement négociées entre entreprises et syndicats, même lorsqu'elles sont contraires aux droits humains, et par des restrictions à la capacité des tribunaux d'interpréter et d'établir une jurisprudence dominante (article 8 de la loi 13467/2017).

Malgré les inconstitutionnalités non admises dans un Etat démocratique de droit, le gouvernement, les médias et même des membres du pouvoir judiciaire mettent la justice du travail sur la sellette. Promue comme bouc expiatoire de la crise ${ }^{4}$, ils souffrent de divers "chantages institutionnels", allant de menaces d'extinction de l'institution à l'instauration de procès administratifs disciplinaires contre les magistrats qui choisissent d'exercer leur pouvoir de contestation des règles inconstitutionnelles ou de respecter les conventions internationales ratifiées par le Brésil.

10 Les syndicats sont affaiblis par la réforme du travail. Bien que l'élargissement des espaces de négociations collectives puisse, en théorie, renforcer l'action syndicale, des modifications du système légal de négociations collectives sont mises en place, avec la limitation des normes collectives pendant un an, interdisant qu'elles ne soient en vigueur plus longtemps et devant attendre une nouvelle négociation (" ultra-activité »). L'élargissement des fonctions dérogatoires des normes légales plus favorables et la primauté donnée aux accords collectifs d'entreprise ont pour effet d'affaiblir le système de négociation ainsi que les syndicats. L'individualisation des rapports de travail est la tendance devant l'inexistence de soutien à la négociation collective, l'affaiblissement des sources de financement des syndicats et leur éloignement des fiscalisations des licenciements individuels (avec la fin des homologations) et des procédures nécessaires pour la promotion d'exemptions collectives. Leur éloignement des dispenses individuelles avec la fin des homologations) et les procédures nécessaires pour la réalisation des licenciements collectifs.

11 Dans un marché reformulé par des règles qui institutionnalisent le travail informel ou précaire de courte durée, sans garantie et très faiblement rémunéré, ces dernières augmentent le turn-over et l'obsolescence programmée de contrats de travail fabriqués pour ne pas durer.

12 La réforme du travail autorise l'embauche de travailleurs intermittents sans leur garantir la perception d'un salaire minimum par mois ; elle légalise la journée de 12 heures (suivie d'un repos de $36 \mathrm{~h}$, pratique commune dans le secteur de la santé), permet que les femmes enceintes travaillent dans des endroits insalubres. En outre, elle supprime du temps rémunéré les heures de déplacement pour rejoindre le lieu de travail (in itinere) lorsque celui-ci est éloigné de même que tous les moments pendant la journée où l'individu doit passer du temps à se préparer pour exercer sa fonction (temps passé au vestiaire, etc. si jamais l'individu peut se changer ailleurs que dans l'entreprise). Avec l'institutionnalisation de mécanismes informels et précaires les perspectives d'intégration sociale par le travail et les fonctions de réduction des inégalités, de redistribution des revenus et de contrôle des pouvoirs économiques, qui sont l'attribut du droit du travail, entrent dans une crise profonde. Les lois 13429 et 13469/2017 prétendent promouvoir une inversion paradigmatique dans la hiérarchie des règles, en réduisant les droits alors qu'en même temps elles augmentent les devoirs des employés et les pouvoirs des employeurs. 

travail et de formalisation de l'emploi protégé, des années 2004 à 2014, reculent. Selon les statistiques officielles de l'IBGE (Institution brésilien de géographie et de statistiques), le chômage a atteint $12,4 \%$, en moyenne, au $3^{\mathrm{e}}$ trimestre compris entre juillet et septembre 2017, touchant 13 millions de personnes. Des 91,3 millions de personnes occupées dans la période, 33,3 ont leur livret de travail ${ }^{5}$ signé, 22,9 millions travaillent à leur compte et 10,9 millions sont occupées dans des emplois sans livret de travail signé et ne sont pas du tout protégées.

14 Les évidences empiriques ne justifient pas le lien entre le changement de la loi et l'augmentation des postes de travail comme l'a annoncé la propagande en faveur de la réforme. La corrélation entre croissance économique, investissements et politiques publiques en faveur du développement et de l'augmentation du nombre de postes de travail et de la masse salariale va dans la direction opposée à celle suivie par les politiques d'austérité. En effet, ces dernières s'accompagnent de déflation en raison de la diminution des salaires, de la réduction des droits sociaux et de ceux liés au travail et du rétrécissement de la taille de l'Etat.

La réforme du travail brésilienne suit à la lettre l'exemple d'un mariage parfait entre les politiques d'austérité ultralibérales et le retour au pouvoir des élites conservatrices d'origine esclavagiste. Comme le dit Mark Blyth (2013: 32), de telles politiques économiques se nourrissent d'illusions statistiques et redistributives et ont des effets différenciés sur les divers segments sociaux: peu sont invités à la fête mais nous sommes tous invités à en payer l'addition, avec une facture qui est bien plus élevée pour qui se situe au bas de l'échelle sociale. Au Brésil, cependant, c'est encore pire que cela. Comme l'exprimait le poète Cazuza : «à la majeure partie qui n'est pas invitée, il ne restera que le plaisir de garer les voitures ", i.e. d'être voiturier.

\section{BIBLIOGRAPHIE}

BLYTH, Mark, Austeridade. A história de uma ideia perigosa., Lisboa, Quetzal editores, 2013.

ESPING-ANDERSEN, Gosta, As três economias políticas do Welfare State, Lua Nova , 1991, n²4, p. 85-116, http://www.scielo.br/scielo.php? script=sci_arttext\&pid=S0102-64451991000200006\&lng=en\&nrm=iso, page consultée le 6 novembre 2017.

\section{NOTES}

1. Proposition d'amendement à la Constitution, $\mathrm{n}^{\circ} 247$ de 2016 qui repousse l'âge minimum de la retraite, limite les pensions et valeurs à recevoir au titre de l'inactivité.

2. La Consolidação das Leis do Trabalho (CLT) est une loi de 1943 portant sur le droit du travail et le droit processuel du travail. C'est le pilier sur lequel reposent les relations professionnelles formelles au Brésil (ndt).

IdeAs, 10 | 2017 
3. La Mesure provisoire $n^{\circ} 805$ du 30 octobre 2017 repousse ou supprime les augmentations de salaire des fonctionnaires fédéraux pour les exercices à venir.Entre autres règles de précarisation du travail, on note le Décret 9127 de 2017, qui élargit l'autorisation du travail le dimanche et jours fériés pour le commerce de détail et du transport qui s'y rapporte, sans se soucier des syndicats. La Medida Provisória (MP) est un instrument qui a force de loi, adopté par le président de la République, pour des cas d'urgence (ndt).

4. Expression utilisée diverses fois par le président de l'Association nationale des juges du travail - Anamatra, Guilherme Guimarães Feliciano, pour démontrer les influences indues qui cherchent à contraindre les juges du travail à ne pas exercer leur contrôle de la constitutionnalité des règles, sans les interpréter.

5. Sorte de passeport dans lequel sont consignés tous les emplois occupés par l'individu.

\section{AUTEURS}

\section{SAYONARA GRILLO COUTINHO LEONARDO DA SILVA}

Sayonara Grillo Coutinho Leonardo da Silva est professeure de Droit à l'Université fédérale de Rio de Janeiro - UFRJ et magistrate au Tribunal régional du travail de la 1ère Région. Docteur en droit de l'Université catholique pontificale de Rio de Janeiro (PUC-Rio). E-

mail :sayonara@direito.ufrj.br 